\title{
Haemodynamic monitoring: Cardiovascular system function
}

"Avoid hypotension and hypoxia!" This admoni-
tion came from internists when patients with heart
disease were referred for surgery. The advice was
irritating and wasted because the pioneers in anaes-
thesia had already appreciated the importance of
maintaining cardiovascular and respiratory system
stability during anaesthesia. Monitoring however
was limited to blood pressure, pulse rate, respira-
tion, breath sounds, eye signs and skin colour.

Anaesthetic morbidity and mortality are decreasing in patients with heart disease because safer anaesthetic drugs are now available, but more so because we have acquired the capability to measure physiological parameters which reflect more accurately cardiovascular system function.

The purpose of this review is to emphasize how cardiovascular system function can best be monitored in the perioperative period. We will examine: who is at risk for cardiovascular decompensation, and what is the minimal information monitoring must provide to ascertain that cardiovascular performance is adequate for total body requirements.

\section{Assessment of risk}

Patients with coronary artery disease may be at great risk of morbidity and mortality from anaesthesia and surgery and can be assessed conventionally or physiologically.

\section{Conventional assessment}

Coronary artery disease is endemic and many patients may have severe unrecognized coronary disease. These patients may appear physiologically sound and consequently the risk of surgery may be underestimated. This is in contrast to the patient who is known to have had a myocardial infarction (MI), in which case an increased surgical risk is clearly present.

\section{J. Earl Wynands MD FRCP(C)}

\section{MYOCARDIAL INFARCTION}

Numerous studies have established the increase in risk associated with a history of MI. Tarhan et al. in a retrospective study (1967-68) found that a history of a previous MI increased the risk of a perioperative MI fifty-fold. ${ }^{1}$ Patients without a previous MI had a 0.13 per cent rate of infarction, compared to 6 per cent in patients with a history of a previous MI. There was a 37 per cent reinfarction rate when surgery was within three months of an MI, which fell to 16 per cent in the 4-6 month time period and thereafter was stable at 5 per cent. The mortality from reinfarction was 54 per cent. Operations on the thorax and upper abdomen were followed by three times as many reinfarctions as operations at other sites. Reinfarctions were more common on the third postoperative day. Steen et al. repeated the study (1974-75) in the same institution and found that there had been no significant change. ${ }^{2}$ The $0-3$ month reinfarction rate was 27 per cent and the 4-6 month rate was 11 per cent. This failure to show improvement in reinfarction rates, which we believe was general and not confined to their institution, was surprising because anaesthetists, by the time of the second study, were aware of the importance of maintaining the myocardial oxygen balance. This study, however, did show that the risk of surgery in the face of a recent MI was significantly increased if the patient had hypertension, diabetes, persistent angina, emergency surgery, surgery lasting more than five hours or hypotension (more than 30 per cent below control).

Rao et al. studied reinfarctions prospectively

From the Departments of Anaesthesia and Surgery, McGill University and Royal Victoria Hospital, 687 Pine Avenue West, Montreal, Quebec H3A IAI. 
(1977-82) and reported a dramatic improvement. ${ }^{3}$ The reinfarction rate when surgery was within 3 months of an MI was 5.7 per cent and 2.3 per cent in the 4-6 month interval. They attributed this improvement to preoperative optimization of the patient's status, aggressive invasive haemodynamic monitoring and prompt treatment of any haemodynamic aberration. The aggressive monitoring and treatment was continued until the fourth postoperative day. The success of this approach must be confirmed by other centres. If confirmed this management protocol should markedly improve reinfarction rates that have stood for decades.

\section{MULTIPLE RISK FACTORS}

Kennedy reported the results of a collaborative study on risk factors in patients undergoing coronary artery surgery. ${ }^{4}$ Risk was increased if the patient had left main coronary artery disease, triple vessel or greater disease, venticular aneurysm, low ejection fraction, heart failure, diabetes, hypertension, or was aged and female.

Paiement et al. examined eight risk factors in patients having open heart surgery and correlated them with mortality: (1) poor left ventricular function, (2) congestive heart failure, (3) unstable angina or recent (less than six weeks) MI, (4) age over 65 years, (5) severe obesity (Body Mass Index $>30$ ), (6) reoperation, (7) emergency surgery, (8) other significant or uncontrolled systemic disturbances. Patients with none of the risk factors had a 0.4 per cent mortality, those with one factor had 3.1 per cent mortality and those with two or more 12.5 per cent. 5

Although these studies specifically applied to cornary artery surgery, I believe they also apply to general surgical patients with coronary artery disease.

\section{GOLDMAN RISK INDEX}

Goldman, in a retrospective study of 1,001 patients, developed a scoring system for cardiac risk factors. ${ }^{6}$ Nine independent significant correlates of serious cardiac complications were identified. Points were assigned to each of the correlates as listed in Table I. Patients were divided into four classes (Table II) depending on the number of points they accumulated and morbidity and mortality predicted.
TABLE I Goldman index risk factors ${ }^{6}$

\begin{tabular}{lc}
\hline Factor & Score \\
\hline Signs of congestive heart failure & 11 \\
Myocardial infarction in the past 6 months & 10 \\
Premature ventricular beats (>5/min) & 7 \\
Other than sinus rhythm & 7 \\
70 or more years old & 5 \\
Emergency surgery & 4 \\
Vascular, intrathoracic or upper abdominal surgery & 3 \\
Aortic stenosis & 3 \\
Poor general condition & 3 \\
Total & $\frac{3}{53}$ \\
\hline
\end{tabular}

Some believe that the Goldman index underestimates risk, but nevertheless it is a scoring system that identifies factors which may be altered by treatment and reduce risk. It is also a valuable reference to use when a surgical consultation asks us to assess risk in view of the patient's medical status. It is also possible that the Goldman Risk Index considerably overestimates morbidity and mortality, if the results and recommendations of Rao et al. quoted above are confirmed. When a patient does not have a history of previous MI and is class III or IV on the Goldman Index, a high degree of suspicion of advanced coronary artery disease should exist if the patient has risk factors such as familial history, cigarette smoking, diabetes, hypertension, high plasma concentrations of low density lipoproteins, Type A personality, etc.

\section{Physiological assessment}

Shibutani and Del Guercio believe that many patients over the age of 65 have unrecognized severe compromised cardiac function and impaired

TABLE II Goldman cardiac risk index ${ }^{6}$

\begin{tabular}{lclll}
\hline Class & $\begin{array}{l}\text { No or only } \\
\text { rotal }\end{array}$ & $\begin{array}{l}\text { Life* } \\
\text { minor } \\
\text { complications }\end{array}$ & $\begin{array}{l}\text { complications } \\
\text { coming }\end{array}$ & $\begin{array}{l}\text { Cardiac } \\
\text { deaths }\end{array}$ \\
\hline I & $0-5$ & $99 \%$ & $0.7 \%$ & $0.2 \%$ \\
II & $6-12$ & $93 \%$ & $5 \%$ & $2 \%$ \\
III & $13-25$ & $86 \%$ & $11 \%$ & $2 \%$ \\
IV & 26 & $22 \%$ & $22 \%$ & $56 \%$ \\
\hline
\end{tabular}

*Documented intraoperative or postoperative myocardial infarction, pulmonary oedema or ventricular tachycardia without progression to cardiac death. 
oxygen delivery which can only be identified by aggressive invasive monitoring. ${ }^{7}$ This approach makes it possible to estimate myocardial efficiency by measuring cardiac output, left and right sided filling pressures and then constructing Starling curves. Systemic vascular resistance, pulmonary vascular resistance and left and right stroke work indices can be calculated. Finally, oxygen delivery and utilization can be measured and the total efficiency of the cardiovascular system estimated.

They studied preoperatively 148 elderly patients cleared for surgery by conventional assessment. Only 13.5 per cent had normal measured haemodynamic, respiratory and oxygen transport function. Mild physiologic aberration not requiring a delay in surgery, or more severe abnormalities indicative of high operative risk were found in 63.5 per cent of the patients. These patients had the identified deficits appropriately treated and proceeded safely through surgery. Major functional deficits occurred in 23 per cent and could not be corrected. It was recommended that surgery be cancelled for these patients: When this warning was disregarded all the patients who underwent surgery died.

VENTRICULAR FUNCTION AND FILLING PRESSURES Elderly patients may have reduced ventricular compliance and this is certainly true in the presence of severe ischemic heart disease and MI. Optimal ventricular performance may require a left ventricular end diastolic pressure (LVEDP) between $1.97 \mathrm{kPa}$ $(15 \mathrm{mmHg})$ and $3.28 \mathrm{kPa}(25 \mathrm{mmHg}){ }^{8,9}$ If we follow the recommendations of Shibutani and Del Guercio, all elderly patients scheduled for major surgery would have preoperative invasive monitoring with pulmonary and peripheral artery catheters.

A significant number of patients may have a normal central venous pressure (CVP) $(0.66 \mathrm{kPa}-$ $5 \mathrm{mmHg})$, and LVEDP of $1.3 \mathrm{kPa}(10 \mathrm{mmHg})$ but have a low cardiac output $(\mathrm{CO})$ of $1.9 \mathrm{~L} \cdot \mathrm{m}^{-2}$. Their measured oxygen delivery would be low and, because blood pressure is within normal limits, the systemic vascular resistance (SVR) would be high. The aim of treatment is to increase $\mathrm{CO}$, decrease SVR and increase oxygen delivery while maintaining systemic blood pressure and heart rate within normal limits. The first therapeutic intervention is a fluid challenge which will increase the LVEDP.
Successive challenges are given until an LVEDP is attained which produces a normal cardiac output $\left(3 \mathrm{~L} \cdot \mathrm{m}^{-2}\right)$. Care must be taken, however, to avoid left ventricular failure. If an LVEDP of $2.63 \mathrm{kPa}$ ( $20 \mathrm{mmHg}$ ) is produced and the $\mathrm{CO}$ remains low, it may be improved by reducing SVR with a vasodilator. If these two interventions do not produce normal haemodynamics, an inotropic drug is indicated. When cardiovascular system function and oxygen delivery are normalized, the patients are ready for surgery.

This approach to the elderly population is attractive, particularly if they are to have major surgery. It identifies patients who are at risk when they may have been declared low risk by conventional assessment. It does, however, present great logistic problems. Many centres may not have the physical capabilities for duplicating Shibutani's preoperative assessment. The intensive invasive monitoring must be done in specialized areas by physicians trained to interpret haemodynamic data.

If we are to duplicate Rao's results in patients with a history of $\mathrm{MI},{ }^{3}$ all hospitals must have the capability of invasive monitoring. The only presently acceptable compromise to this logistic problem is for anaesthetists to insert invasive lines prior to the induction of anaesthesia in all patients conventionally assessed as high risk for surgery. These patients will have their base line haemodynamic profile determined and, if judged inadequate for surgery, appropriate interventions can be made and the surgical risk decreased. The intensive monitoring initiated in the operating room should be continued in the recovery room for an appropriate period to verify good cardiac performance and axygen delivery.

\section{An approach to monitoring}

Significant advances have been made in monitoring capabilities, both invasive and non-invasive. We cannot explore here all methods of cardiovascular system monitoring, pointing out their advantages and limitations; they have been reviewed elsewhere. ${ }^{10-12}$

Perioperative myocardial ischemia, which can cause myocardial dysfunction, impaired oxygen delivery and MI, may be difficult to diagnose. This is evident from the numerous modifications of ECG leads recommended for the detection of myocardial 
ischemia and the search for other indicators such as 2-D echocardiography, pulmonary capillary wedge pressure wave analysis, endocardial viability ratios and nuclear scanning devices. The difficulty in diagnosis exists because severe ischemia may be limited to a specific region of the heart, e.g. inferior wall of the left ventricle, and the ECG lead employed have inadequate specificity and sensitivity to detect the ischemic area. We will not attempt to resolve the problems related to the detection of myocardial ischemia but will refer the reader to other reviews ${ }^{13.14}$ Information derived from a pulmonary artery catheter may be indispensible in optimizing cardiovascular system performance and is dealt with in the third section of this review.

All patients must have cardiovascular system function monitored by continuous ECG and intermittent recording of blood pressure. Many believe that an esophageal stethoscope is mandatory because it gives constant information on breath sounds, the rate and regularity of the heart beat and, by changes in intensity of heart sounds, indicates changes in $\mathrm{CO}$. When faced with patients who are at risk of developing cardiac complications the intensity of monitoring must be increased, depending on the anticipated risk.

\section{Intra-arterial monitoring}

The monitoring of systemic blood pressure will be extensively covered in the second section of this review, but we wish to emphasize the following points.

We find continuous arterial blood pressure monitoring indispensible in dealing with high risk patients who are undergoing major surgery, although new methods of intermittent non-invasive blood pressure monitoring may make the use of intra-arterial continuous monitoring unnecessary in many cases. However, the continuous display of a reliable beat-to-beat blood pressure is extremely valuable in patients with threatened or existing cardiac dysfunction. In addition to showing the blood pressure, the pulse wave contour is a crude but valuable indicator of myocardial contractility. The arterial catheter facilitates the taking of samples for blood gas analysis. A 20-gauge nontapered teflon catheter placed in a radial artery has few serious complications. ${ }^{15}$ The transduced pressure from the artery, to be accurate must accom- modate strict criteria for leveling, calibrating, dampening, overshoot, etc. ${ }^{16}$

\section{Oxygen transport system}

The amount of oxygen delivered to the tissues per minute depends on the $\mathrm{CO}$ and the oxygen content of arterial blood $\left(\mathrm{CaO}_{2}\right)$. The latter is related to haemoglobin concentration, its saturation and a small amount of oxygen dissolved in plasma.

$$
\begin{aligned}
\mathrm{CaO}_{2}= & \mathrm{Hb} \mathrm{gm} / \mathrm{dl} \times 1.36 \\
& \times \mathrm{Hb} \text { per cent saturation }+\mathrm{PaO}_{2} \times 0.003
\end{aligned}
$$

The normal value of $\mathrm{CaO}_{2}$ is $19-20$ volumes per cent so that a $\mathrm{CO}$ of five litres would deliver $1,000 \mathrm{cc}$ of oxygen to the tissues per minute. The oxygen consumption per minute $\left(\mathrm{VO}_{2}\right)$ approximates 250 cc. This 250 cc oxygen extraction is 25 per cent of the oxygen delivery and, ignoring the dissolved oxygen, means venous oxygen content will have been reduced by 25 per cent and thus venous haemoglobin saturation will be 75 per cent. This will be associated with a venous $\mathrm{PvO}_{2}$ of $5.26 \mathrm{kPa}$ ( $40 \mathrm{mmHg}$ ). The venous oxygen content constitutes venous oxygen reserve which is only used under exceptional circumstances. Under normal conditions a decrease in $\mathrm{CaO}_{2}$ or an increase in $\mathrm{VO}_{2}$ is compensated for by an increase in $\mathrm{CO}$ and the venous stores are untouched. When $\mathrm{CO}$ cannot compensate for decreased $\mathrm{CaO}_{2}$ or meet the increases in $\mathrm{VO}_{2}$, the venous oxygen reserve will be used. When $\mathrm{SvO}_{2}$ or $\mathrm{PvO}_{2}$ fall below normal, there is an imbalance between tissue oxygen supply and demand. In patients with heart disease this is often due to a decrease in $\mathrm{CO}$.

\section{Measurement of $\mathrm{SvO}_{2}$}

A pulmonary artery catheter is available which, in addition to the usual measurement capacity, provides the ability to continuously monitor $\mathrm{SvO}_{2}$. (Oximetrix Shaw ${ }^{\mathrm{TM}}$ catheter oximeter system; Oximetrix Inc., Mountain View, Calif.). Three light beams of different wave lengths are passed down a monofilament of the fiberoptic catheter. The light is refracted off hemoglobin and passes by a second monofilament to a photosensor. Oxyhemoglobin and reduced haemoglobin have different light absorption characteristics. The detected light is converted to electrical signals, analysed by a signal 
processor and average percentage haemoglobin saturation for the previous five seconds computed and displayed.

\section{Interpretation of $\mathrm{SvO}_{2}$}

When the $\mathrm{SvO}_{2}$ falls below normal it indicates that oxygen delivery has not been adequate to meet tissue demands. In a given patient it does not indicate whether the mismatch is due to decreased $\mathrm{CO}$, a low $\mathrm{CaO}_{2}$ or an increased $\mathrm{VO}_{2}$. However, in certain situations as $\mathrm{MI}$ or following aorto-coronary bypass surgery it may have a high correlation with a low $\mathrm{CO}$

When neurolept anaesthesia is used for aortocoronary bypass surgery there is approximately a 22 per cent fall from normal in $\mathrm{VO}_{2}$ to $108 \mathrm{ml} / \mathrm{min} / \mathrm{m}^{2}$ which remains constant as long as oxygen delivery exceeds $330 \mathrm{ml} / \mathrm{min} / \mathrm{m}^{2} ;{ }^{17}$ below this figure $\mathrm{VO}_{2}$ will fall. Between $330 \mathrm{ml} / \mathrm{min} / \mathrm{m}^{2}$ and the normal $550 \mathrm{ml} / \mathrm{min} / \mathrm{m}^{2}$ there will be a fall in the $\mathrm{SvO}_{2}$ but the magnitude of the fall may not be proportional to the decline of oxygen supply as vasoregulation, if intact, will have diverted blood flow to essential organs. When $\mathrm{VO}_{2}$ begins to fall it is a sign that lactic acid is being produced in some regional circulatory beds. It has been shown that a venous $\mathrm{PO}_{2}$ of 28 is associated with lactic acid production, which heralds a poor prognosis. ${ }^{18}$ Haemoglobin is 50 per cent saturated at a $\mathrm{PO}_{2}$ of $3.42 \mathrm{kPa}(26 \mathrm{mmHg})$, thus an $\mathrm{SvO}_{2}$ below 50 per cent signals the beginning of regional lactic acid production. An $\mathrm{SvO}_{2}$ of 60 per cent lies approximately halfway between normal and the point at which lactic acid production begins. Clinicians treating patients who have had an MI feel that an $\mathrm{SvO}_{2}$ of 60 per cent that does not improve in spite of appropriate interventions is associated with a poor prognosis ${ }^{7}$ and this also may apply to patients in the perioperative period. From the above we may conclude that $\dot{\mathrm{VO}}_{2}$ may fall in the anaesthetized patient when the oxygen delivery falls below $330 \mathrm{ml} / \mathrm{min} / \mathrm{m}^{2}$ and lactic acid production may begin. This will be reflected by an $\mathrm{SvO}_{2}$ of 50 per cent or lower which must be raised to a more acceptable value ( 65 per cent) by investigating and correcting the cause of the tissue oxygen imbalance. Problems may be encountered postoperatively which are refiected by the high incidence of a recurrent $\mathrm{MI}$ on the third postoperative day. ${ }^{1} \mathrm{VO}_{2}$ may be increased at this time, ncessitating more than the $330 \mathrm{ml} / \mathrm{min} / \mathrm{m}^{2}$ oxygen delivery that was adequate to prevent lactic acid production in the operating room and the $\mathrm{SvO}_{2}$ may be the first indication that a patient has an inadequate oxygen delivery to meet tissue demands. In patients undergoing cardiac surgery, a low $\mathrm{SvO}_{2}$ has been related to many causes which responded to appropriate therapy. ${ }^{19} \mathrm{We}$ believe that $\mathrm{SvO}_{2}$ is an extremely valuable early warning sign of a derangement in whole body oxygen balance. Returning $\mathrm{SvO}_{2}$ to normal by an appropriate intervention will reduce perioperative morbidity and mortality in patients at high risk from cardiac disease.

\section{References}

1 Tarhan S, Moffut EA, Taylor WF, et al. Myocardial infarction after general anesthesia. JAMA 1972; 220: 1451-4.

2 Steen PA, Tinker JH, Tarhan S. Myocardial reinfarction after anesthesia and surgery. JAMA 1978; 239: $2566-70$.

3 Rao TLK, Jacobs KH, El-Etr A. Reinfarction following anesthesia in patients with myocardial infarction. Anesthesiology 1983; 59: 499-505.

4 Kennedy JW, Kaiser GC, Fisher LD, et al. Clinical and angiographic predictors of operative mortality from the collaborative study in coronary artery surgery (CASS). Circulation 1981; 63: 793-802.

5 Paiement B, Pelletier C, Dyrda I, et al. A simple classification of the risk in cardiac surgery. Can Anaesth Soc J 1983; 30: 61-8.

6 Goldman L, Caldera DL, Nussbaum SR et al. Multifactorial index of cardiac risk in noncardiac surgical procedures. N Engl J Med 1977; 297; 845-50.

7 Shibutani K, Del Guercio LRM. Preoperative hemodynamic assessment of the high-risk patient. Seminars in Anesthesia 1983; 2: 231-40.

8 Crexalls $C$, Chatterjee $K$, Forrester J, et al. Optimal level of filling pressure in the left side of the heart in acute myocardia] infarction. $\mathrm{N}$ Engl $\mathrm{J}$ Med 1973; 289: 1263-6.

9 Broder MI, Cohn JN. Evolution of abnormalities in left ventricular function after acute myocardial infarction. Circulation 1972; 46: 731-43.

10 Matthay MA. Invasive hemodynamic monitoring in critically ill patients. Clinics in Chest Medicine 1983; 4: 233-49. 
11 Wiedemann HP, Matthay MA, Matthay RA. Cardiovascular-pulmonary monitoring in the intensive care unit (Part 1). Chest 1984; 85: 537-49.

12 Wiedemann HP, Matthay MA, Matthay RA. Cardiovascular-pulmonary monitoring in the intensive care unit (Part 2). Chest 1984; 85: 656-68.

13 Griesemer $R W$, Kaplan JA. Myocardial ischemia during non-cardiac surgery: diagnosis and treatment. Seminars in Anesthesia 1982; 219-25.

14 Rao TLK. Cardiac monitoring for the noncardiac surgical patient. Seminars in Anesthesia 1983; 2 : 241-50.

15 Goldenheim PD, Kazemi $H$. Cardiopulmonary monitoring of critically ill patients (Part 2), N Engl J Med 1984; 311: 776-80.

16 Snyder JV, Carroll GC. Tissue oxygenation: A physiological approach to a clinical problem. Current Problems in Surgery 1982; 19: 653-719.

17 Shibutani K, Komatsu T, Kubal K, et al. Critical level of oxygen delivery in anesthelized man. Crit Care Med 1983; 11: 640-3.

18 Kasnitz $P$, Druger GL, Yorra F, et al. Mixed venous oxygen tension and hyperlactatemia. JAMA 1976, 236: 570-4.

19 Jamieson WRE, Turnbull KW, Larrieu AJ, et al. Continuous monitoring of mixed venous oxygen saturation in cardiac surgery. Can J Surg 1982; 25 : $538-43$. 